\title{
Considerations when measuring myocardial perfusion reserve by cardiovascular magnetic resonance using regadenoson
}

Nicole M Bhave, Benjamin H Freed, Chattanong Yodwut, Denise Kolanczyk, Karin Dill, Roberto M Lang, Victor Mor-Avi and Amit R Patel

\begin{abstract}
Background: Adenosine cardiovascular magnetic resonance (CMR) can accurately quantify myocardial perfusion reserve. While regadenoson is increasingly employed due to ease of use, imaging protocols have not been standardized. We sought to determine the optimal regadenoson CMR protocol for quantifying myocardial perfusion reserve index (MPRi) - more specifically, whether regadenoson stress imaging should be performed before or after rest imaging.

Methods: Twenty healthy subjects underwent CMR perfusion imaging during resting conditions, during regadenoson-induced hyperemia $(0.4 \mathrm{mg})$, and after $15 \mathrm{~min}$ of recovery. In 10/20 subjects, recovery was facilitated with aminophylline $(125 \mathrm{mg})$. Myocardial time-intensity curves were used to obtain left ventricular cavity-normalized myocardial up-slopes. MPRi was calculated in two different ways: as the up-slope ratio of stress to rest (MPRi-rest), and the up-slope ratio of stress to recovery (MPRi-recov).

Results: In all 20 subjects, MPRi-rest was $1.78 \pm 0.60$. Recovery up-slope did not return to resting levels, regardless of aminophylline use. Among patients not receiving aminophylline, MPRi-recov was $36 \pm 16 \%$ lower than MPRi-rest $(1.13 \pm 0.38$ vs. $1.82 \pm 0.73, P=0.001)$. In the 10 patients whose recovery was facilitated with aminophylline, MPRi-recov was $20 \pm 24 \%$ lower than MPRi-rest (1.40 \pm 0.35 vs. $1.73 \pm 0.43, P=0.04)$, indicating incomplete reversal. In 3 subjects not receiving aminophylline and 4 subjects receiving aminophylline, up-slope at recovery was greater than at stress, suggesting delayed maximal hyperemia.

Conclusions: MPRi measurements from regadenoson CMR are underestimated if recovery perfusion is used as a substitute for resting perfusion, even when recovery is facilitated with aminophylline. True resting images should be used to allow accurate MPRi quantification. The delayed maximal hyperemia observed in some subjects deserves further study.
\end{abstract}

Trial registration: ClinicalTrials.gov NCT00871260

Keywords: Stress CMR, Regadenoson, Vasodilators

\footnotetext{
* Correspondence: amitpatel@uchicago.edu

Departments of Medicine and Radiology, University of Chicago, Cardiac Imaging Center, 5841 S. Maryland Ave., MC5084, Chicago, IL 60637, USA
} 


\section{Background}

In recent years, vasodilator stress cardiovascular magnetic resonance (CMR) perfusion imaging has been shown to be a sensitive and specific means of diagnosing coronary artery disease [1-9]. CMR also offers a wealth of data regarding myocardial structure and function, without exposing patients to ionizing radiation, and aids in risk stratification for future adverse cardiovascular events [10-12]. Because of these advantages, vasodilator CMR is a rapidly burgeoning methodology.

Most commonly, vasodilator stress CMR is performed with adenosine. This drug requires a continuous infusion, such that separate IV lines are required for vasodilator and contrast agent. Due to activation of $\mathrm{A}_{1}, \mathrm{~A}_{2 \mathrm{~B}}$ and $\mathrm{A}_{3}$ receptors, adenosine has a variety of undesirable side effects, which include atrioventricular (AV) block, hypotension, and bronchospasm [13]. These occurrences can interrupt workflow and, in rare circumstances, compromise patient safety [14]. Regadenoson, a selective $\mathrm{A}_{2 \mathrm{~A}}$ receptor agonist, is an appealing alternative for stress CMR because it is administered as a single, standard-dose bolus (such that only one IV line is required) and has a more favorable side-effect profile $[15,16]$. We have demonstrated the feasibility and safety of regadenoson CMR and have shown that perfusion defects on stress CMR images predict future need for revascularization [17]. One advantage of stress CMR is the ability to quantify myocardial perfusion reserve (MPR), which is less dependent upon interpreter expertise and improves the accuracy of the detection of multivessel coronary artery disease [18]. Others have shown that MPR obtained by vasodilator stress CMR is similar, regardless of whether hyperemia is induced with adenosine or regadenoson [19].

Because the half-life of adenosine is $2-10$ seconds [16], and thus the hyperemic effects of the drug are expected to be completely resolved after 10-15 minutes, stress CMR imaging with adenosine is often performed before rest imaging [10,20,21]. This stress followed by rest approach eliminates the possibility that stress images could be contaminated by delayed enhancement from previously administered gadolinium-based contrast, potentially masking the presence of perfusion defects.

Clinicians often perceive regadenoson as a short-acting agent because its side effects are short-lived, but the drug has a terminal half-life of approximately 2 hours [22]. After administration, it redistributes rapidly throughout the body, followed by slower elimination. Due to regadenoson's longer half-life, recovery is occasionally facilitated with aminophylline before resting images are acquired $[17,23]$. However, the residual impact of regadenoson on coronary blood flow during the clearance period is not known.

Accordingly, the goals of this study were: (1) to determine whether a stress-recovery regadenoson CMR protocol can reliably quantify myocardial perfusion reserve as compared to a rest-stress protocol, (2) to ascertain whether post-stress aminophylline administration results in a complete return of myocardial perfusion to a pre-stress level, and (3) to establish a reference range for MPR index (MPRi) in normal volunteers.

\section{Methods}

\section{Study subjects}

Twenty healthy volunteers (70\% female, 70\% Caucasian, mean age $32 \pm 10 \mathrm{yr}$ ) were prospectively recruited for vasodilator CMR. Exclusion criteria were: history of coronary artery disease, congestive heart failure, or diabetes mellitus; non-sinus rhythm; any contraindication to regadenoson (including heart block, chronic obstructive pulmonary disease, or severe asthma); and any contraindication to contrast-enhanced CMR (including history of chronic kidney disease with glomerular filtration rate $<30 \mathrm{~mL} / \mathrm{min}$, implantable pacemaker or defibrillator, severe claustrophobia, or adverse reaction to gadolinium-based contrast agents). All subjects were instructed not to consume caffeine for 12 hours prior to the test. A 12-lead ECG was performed in each subject prior to imaging to rule out high-degree AV nodal block. The University of Chicago institutional review board approved the study.

\section{CMR image acquisition protocol \\ Myocardial structure and function}

CMR images were acquired using a 1.5-T scanner (Achieva, Philips, Best, Netherlands) and a 5-element phased array cardiac coil. Retrospectively gated cine images were obtained with a steady-state free precession (SSFP) sequence (TR $2.9 \mathrm{~ms}$, TE $1.5 \mathrm{~ms}$, flip angle $60^{\circ}$, and temporal resolution $\sim 40 \mathrm{~ms}$ ). A stack of short-axis slices ( $8 \mathrm{~mm}$ thickness, $2 \mathrm{~mm}$ gap) from base to apex was acquired. Standard 2-, 3-, and 4-chamber long-axis views were also obtained.

\section{Myocardial perfusion imaging}

A hybrid gradient echo/echo planar imaging sequence (voxel size $\sim 2.57 \times 2.5 \mathrm{~mm}$, slice thickness $10 \mathrm{~mm}$, flip angle $20^{\circ}$, repetition time $5.9 \mathrm{~ms}$, echo time $2.5 \mathrm{~ms}$, EPI factor 5 , delay time $80 \mathrm{~ms}$, and SENSE factor 1.3) was used to acquire short-axis slices at 3 levels of the left ventricle (LV) during first pass of gadobenate dimeglumine $(0.05 \mathrm{mmol} /$ $\mathrm{kg}$ at $4 \mathrm{~mL} / \mathrm{sec}, 15$ patients) or gadolinium-DTPA $(0.025 \mathrm{mmol} / \mathrm{kg}$ at $4 \mathrm{~mL} / \mathrm{sec}, 5$ patients $)$ for 50 consecutive heartbeats. All subjects underwent myocardial perfusion imaging during 3 separate physiologic states, each separated by 15 minutes: (1) resting conditions, (2) 1 minute after regadenoson administration (Lexiscan $0.4 \mathrm{mg}$ IV bolus, Astellas Pharma), and (3) during recovery. In 10 randomly selected subjects, recovery was facilitated with 
aminophylline (125 mg IV), administered 1 minute after stress imaging (Figure 1). Heart rate was monitored continuously, and mean arterial blood pressure was recorded at rest, at peak stress, and during recovery.

\section{Late gadolinium enhancement imaging}

Late gadolinium enhancement (LGE) images with slice positions identical to the above described cines were obtained 5-10 minutes after the third infusion of contrast, using a T1-weighted gradient echo pulse sequence with a phase sensitive inversion recovery reconstruction (TR $4.5 \mathrm{~ms}$, TE $2.2 \mathrm{~ms}$, TI 250-300 ms, flip angle $30^{\circ}$, flip angle $5^{\circ}$, voxel size $2 \times 2 \times 10 \mathrm{~mm}$, SENSE factor 2$)$. Optimal inversion time was chosen based on a TI scout (typically 250-300 ms).

\section{Image analysis}

Images were analyzed with a commercial software package (Philips View Forum, Best, Netherlands). Short-axis cine slices were used to measure ventricular volumes and ejection fractions (LVEF) using the Simpson method of disks. For semi-quantitative perfusion analysis, epicardial and endocardial borders of the mid-LV slice were manually traced for each frame, myocardial segmentation was applied, and time-signal intensity curves were generated for each myocardial segment and the LV cavity. The maximum myocardial up-slopes were normalized to the LV cavity up-slope and averaged for all myocardial segments (Figure 2). MPRi was calculated as both (1) the up-slope ratio of stress to rest (MPRi-rest) and (2) the up-slope ratio of stress to recovery (MPRirecov). Cine, perfusion, and LGE images were visually assessed by a CMR expert (ARP).

\section{Statistical analysis}

Continuous variables were analyzed using student's t-tests (paired for intragroup comparisons, unpaired for intergroup comparisons; equal variances were not assumed). Categorical variables were analyzed with Fisher's exact test.

\section{Results}

Table 1 shows the basic characteristics and hemodynamic data of the two groups of subjects: 10 subjects who received aminophylline and the remaining 10 subjects. All subjects had normal LVEFs (>50\%) and LV end-diastolic volume indices, with no regional wall motion abnormalities, late gadolinium enhancement, or perfusion defects.

In all subjects, regadenoson significantly increased the heart rate above baseline (mean increase $52 \pm 13$ beats per min, $P<0.001)$. Patients receiving aminophylline poststress had a significantly larger fall in heart rate from stress to recovery (mean decrease $48 \pm 11$ vs. $35 \pm 12$ beats per min, $P=0.03$; Table 1 ). Common side effects of regadenoson included dyspnea or difficulty with breath holds (45\%), palpitations (35\%), chest pressure or heaviness (35\%), and flushing (25\%). Two subjects reported dysgeusia immediately following aminophylline administration. Side effects did not cause any significant delays in scanning, and no adverse events occurred.

In all 20 subjects, myocardial perfusion (as estimated by normalized up-slope) increased from rest to stress: mean rest up-slope was $8.26 \pm 2.40$, and stress up-slope was $14.50 \pm 6.21(P<0.001)$. Regardless of aminophylline administration, recovery up-slope was higher than rest up-slope and not significantly different from stress upslope (Table 2). Among patients who did not receive aminophylline, myocardial perfusion up-slope was consistently greater at recovery than at rest (mean increase $68 \pm 54 \%, P<0.01$; figure 3 ). In the aminophyllinefacilitated recovery group, all but 2 subjects had greater perfusion up-slopes at recovery than at rest (mean increase $34 \pm 37 \%, P=0.02$ ).

Mean MPRi-rest for all 20 subjects was $1.78 \pm 0.60$. Regardless of aminophylline administration, MPRi-recov was lower than MPRi-rest, suggesting incomplete recovery from hyperemia. In the non-aminophylline group, mean MPRi-recov was $36 \pm 16 \%$ lower than MPRi-rest $(1.13 \pm 0.38$ vs. $1.82 \pm 0.73, P=0.001)$. In the aminophylline group, mean MPRi-recov was $20 \pm 24 \%$ lower than MPRi-rest $(1.40 \pm 0.35$ vs. $1.73 \pm 0.43, P=0.04$; Table 3$)$. Although the absolute difference between MPRi-rest and MPRi-recov tended to be smaller in the aminophylline group as compared to the non-aminophylline group, this

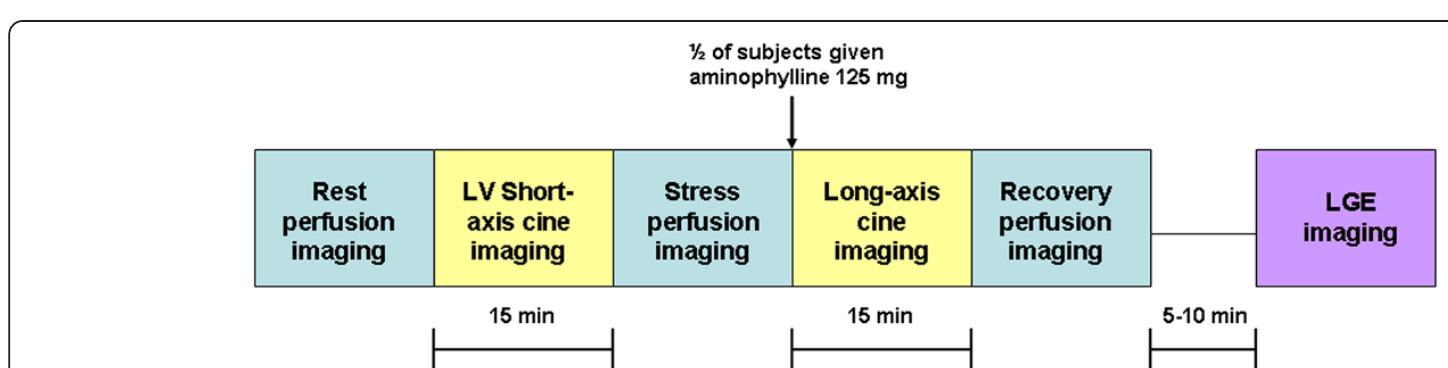

Figure 1 CMR protocol. Perfusion imaging sequences are separated by 15-min intervals as shown. 


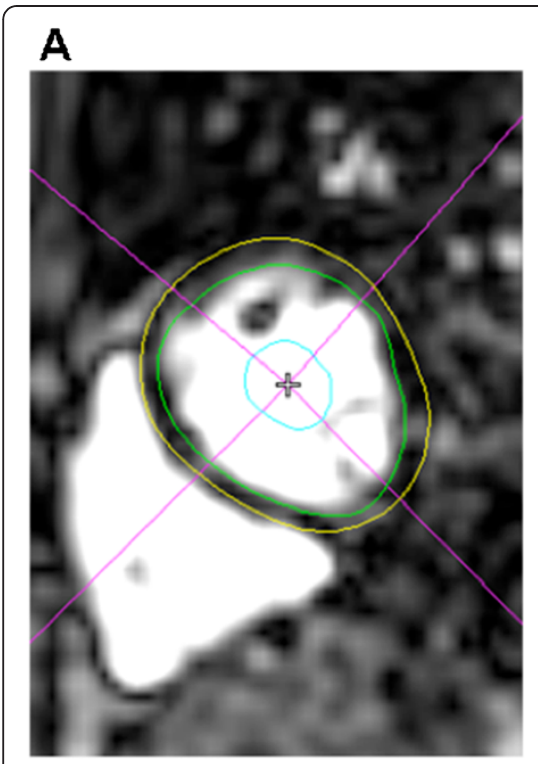

\section{B}

Figure 2 A. Example of myocardial tracing and segmentation in a short-axis slice. Endocardial border is shown in green and epicardial border in yellow. The LV myocardium was segmented into 4 sectors (anterior, lateral, inferior, septal). B. Blood pool and segmental myocardial time-intensity curves. Maximum up-slopes (black lines) were determined for the blood pool and for each myocardial segment; the mean myocardial up-slope was then normalized to the blood pool up-slope.

difference was not statistically significant $(P=0.11$ for intergroup comparison).

Three subjects in the non-aminophylline group and 4 in the aminophylline group had increased myocardial perfusion indices at recovery as compared to stress (Figure 3). These 7 patients exhibited no significant differences in age, body surface area, or gender distribution as compared to all other subjects. Baseline diastolic blood pressure was slightly higher in this group (68 \pm 12 vs. $55 \pm 11 \mathrm{mmHg}, P=0.04$ ). Although subjects in this group tended to have greater heart rate increases at stress, this difference was not statistically significant (56 vs. $50 \mathrm{bpm}, P=0.33$ ). Subjects with more hyperemia at recovery than at stress, by definition, all had MPRirecov $<1$, and MPRi-recov was $43 \pm 11 \%$ lower than MPRi-rest $(0.80 \pm 0.16$ vs. $1.39 \pm 0.10, P<0.001)$. As compared to subjects who were maximally hyperemic at stress, those who were maximally hyperemic at recovery had lower MPRi-rest $(1.39 \pm 0.10$ vs. $1.99 \pm 0.68, P=$ $0.008)$, lower MPRi-recov ( $0.80 \pm 0.16$ vs. $1.52 \pm 0.41, P<$

Table 1 Patient demographics and hemodynamic responses to regadenoson

\begin{tabular}{|c|c|c|c|}
\hline & $\frac{\text { Aminophylline }}{(n=10)}$ & $\frac{\text { No aminophylline }}{(n=10)}$ & $\begin{array}{c}P \\
\text { value }\end{array}$ \\
\hline Age (yr) & $32(7)$ & $32(13)$ & 0.90 \\
\hline$\%$ female & 70 & 50 & 0.65 \\
\hline$\%$ Caucasian & 70 & 50 & 0.65 \\
\hline LVEF (\%) & $61(5)$ & $61(5)$ & 0.97 \\
\hline LVEDV index $\left(\mathrm{mL} / \mathrm{m}^{2}\right)$ & $85(9)$ & $84(14)$ & 0.89 \\
\hline Baseline HR (BPM) & $68(9)$ & $65(14)$ & 0.67 \\
\hline HR change, baseline to stress (BPM) & $+55(11)$ & $+48(13)$ & 0.22 \\
\hline HR change, stress to recovery (BPM) & $-48(11)$ & $-35(12)$ & 0.03 \\
\hline HR change, rest to recovery (BPM) & $+7(9)$ & $+13(12)$ & 0.24 \\
\hline Baseline MAP (mmHg) & $77(11)$ & $78(12)$ & 0.75 \\
\hline Change in MAP, baseline to stress $(\mathrm{mmHg})$ & $+3(10)$ & $+4(7)$ & 0.81 \\
\hline
\end{tabular}

$S D$, standard deviation; $L V E F$, left ventricular ejection fraction; $L V E D V$, left ventricular end-diastolic volume; $H R$, heart rate; $B P M$, beats per minute; $M A P$, mean arterial pressure. Data in parentheses are standard deviations. 
Table 2 Regadenoson CMR perfusion up-slopes at rest, stress, and recovery, with and without aminophylline

\begin{tabular}{lccccc}
\hline & $\begin{array}{c}\text { Rest up-slope } \\
\text { (SD) }\end{array}$ & $\begin{array}{c}\text { Stress up-slope } \\
\text { (SD) }\end{array}$ & $\begin{array}{c}\text { Recovery up-slope } \\
\text { (SD) }\end{array}$ & $\begin{array}{c}\text { P value, recovery vs. } \\
\text { rest }\end{array}$ & $\begin{array}{c}\text { P value, recovery vs. } \\
\text { stress }\end{array}$ \\
\hline $\begin{array}{l}\text { No } \\
\text { Aminophylline }\end{array}$ & $8.79(2.98)$ & $15.99(8.38)$ & $14.08(4.03)$ & $<0.001$ & 0.64 \\
\hline Aminophylline & $7.73(1.64)$ & $13.01(2.53)$ & $10.19(2.98)$ & 0.02 & 0.08 \\
\hline
\end{tabular}

Data in parentheses are standard deviations.

$0.001)$, and higher recovery-to-rest ratios $(1.84 \pm 0.53$ vs. $1.33 \pm 0.35, P=0.05)$. The recovery-to-rest ratios in the delayed hyperemia group were similar to MPRi-rest in the remaining patients $(1.84 \pm 0.53$ vs. $1.99 \pm 0.68$, $P=0.58)$.

\section{Discussion}

Vasodilator stress CMR is being increasingly incorporated into clinical practice for the evaluation of coronary artery disease because of its superior diagnostic performance when compared to single photon emission computed

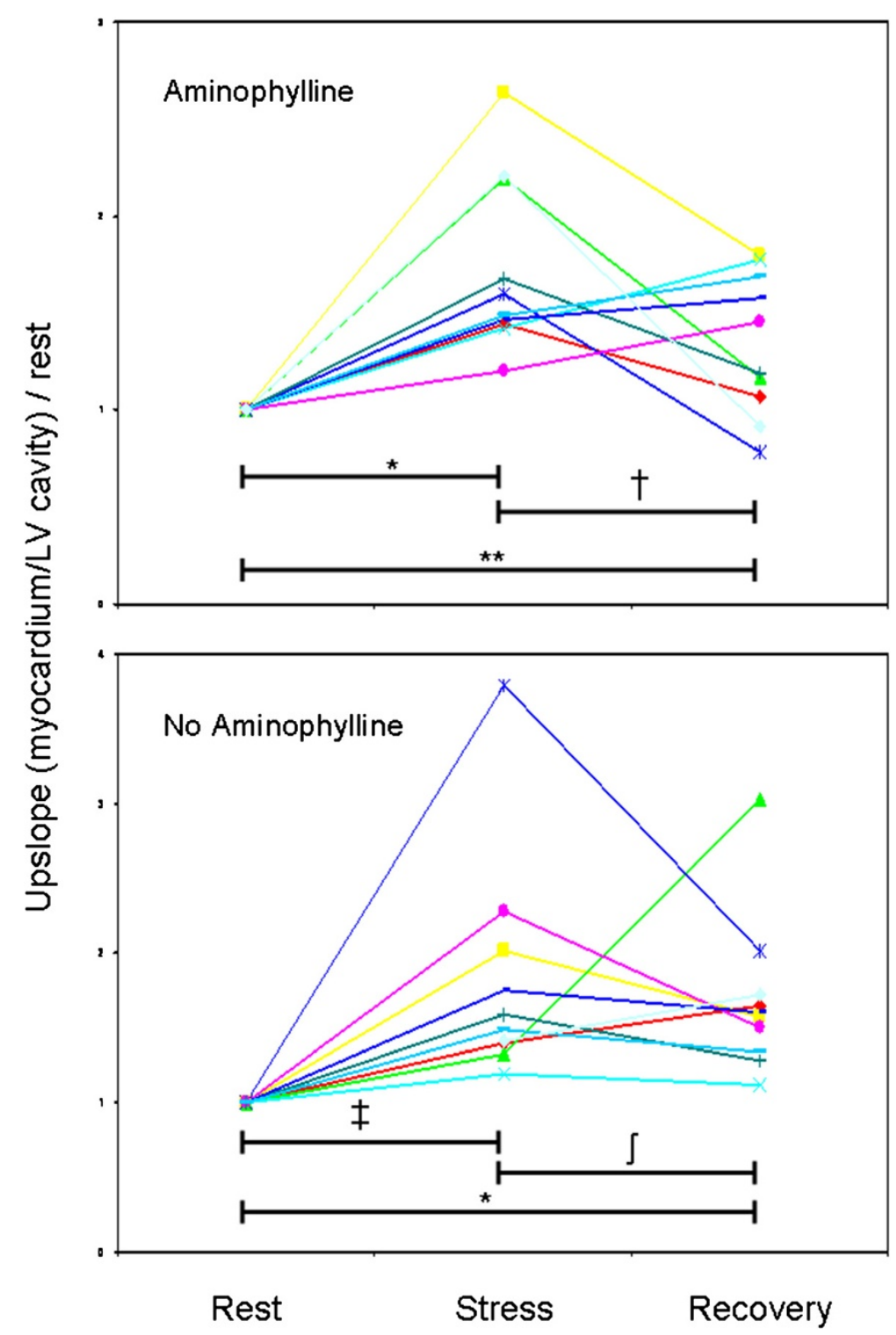

Figure 3 Comparison of perfusion up-slopes at stress and recovery, normalized for rest up-slope, on a per-patient basis. Four subjects in the aminophylline group and 3 subjects in the no-aminophylline group had greater perfusion at recovery than at stress. ${ }^{*} P<0.001 ;{ }^{\dagger} P=0.08$; ${ }^{* *} \mathrm{P}=0.02 ;{ }^{*} \mathrm{P}=0.01 ;{ }^{\int} \mathrm{P}=0.38$. 
Table 3 Comparison of aminophylline and no-aminophylline groups with respect to perfusion reserve

\begin{tabular}{lcccc}
\hline & MPRi-rest, mean (SD) & MPRi-recov, mean (SD) & \% difference (SD) & P value \\
\hline No Aminophylline & $1.83(0.73)$ & $1.13(0.38)$ & 36 & 0.001 \\
\hline $\begin{array}{l}\text { Aminophylline } \\
\text { MPRi-rest, myocardial perfusion reserve index comparing stress to rest; MPRi-recov, myocardial perfusion reserve index comparing stress to recovery. Data in }\end{array}$ \\
$\begin{array}{l}\text { parentheses are standard deviations. } \\
\text { pl.73 }(0.43)\end{array}$
\end{tabular}

tomography (SPECT). Because of its ease of use and improved side-effect profile compared to adenosine, regadenoson is being used more frequently to induce hyperemia during vasodilator stress CMR. However, optimal protocols for regadenoson CMR have not yet been established. In this study, we found that: (1) regadenosoninduced hyperemia persists to a degree, even after 15 minutes of recovery; (2) recovery facilitated with aminophylline only partially reverses the residual regadenoson-induced hyperemia; and (3) some individuals may have delayed maximal hyperemia following the administration of regadenoson. Therefore, when regadenoson myocardial perfusion imaging is performed prior to recovery perfusion imaging, the calculated myocardial perfusion reserve may significantly underestimate the actual myocardial perfusion reserve, irrespective of whether aminophylline is used to reverse hyperemia.

One particular strength of stress CMR is its ability to assess myocardial perfusion, quantitatively or semi-quantitatively, as an adjunct to visual analysis. Assessment of myocardial perfusion reserve has been shown to reduce inter-reader variability and to quantify more accurately the severity of localized ischemia due to epicardial coronary stenosis [24], particularly when ischemia is present in multiple vascular territories [18]. Myocardial perfusion reserve by $\mathrm{CMR}$ can also reveal diffuse ischemia attributable to microvascular dysfunction [25], and is a potentially useful tool for identifying subclinical disease in patients with cardiovascular risk factors [26,27].

We have established, albeit in a relatively small group of normal subjects, a reference range for MPRi-rest with regadenoson, as determined semi-quantitatively using the time-intensity up-slope technique with a hybrid gradient echo/echo planar imaging sequence. Perfusion analysis by this method is rapid enough for routine clinical use and does not require cumbersome dual-bolus imaging, which may be desirable when MPR is determined quantitatively by deconvolution methods [28]. DiBella and colleagues have previously compared perfusion reserve with regadenoson to that with adenosine and found good agreement, using an ultra-fast gradient echo sequence (Turbo FLASH, Siemens) and deconvolution. However, their study included patients with CAD and cardiovascular risk factors, so their findings cannot be used as reference values [19]. Based on our mean MPRi-rest $1.78 \pm 0.60$, we calculate that a 58-patient sample size would be needed to detect a $25 \%$ difference in perfusion reserve between normals and abnormals (80\% power, $\alpha=0.05$ ). Accordingly, in future drug trials aimed at treatment of microvascular dysfunction, regadenoson stress CMR could allow detection of significant differences in perfusion reserve in relatively small cohorts. Our reported normal ranges may also be useful for the development of appropriate semi-quantitative regadenoson stress CMR cut-off values for detecting significant coronary disease and microvascular dysfunction.

Aminophylline is a dissociable complex of theophylline, a methylxanthine adenosine receptor antagonist, and ethylenediamine, which improves solubility. In one small cardiac catheterization study, aminophylline appeared to reverse regadenoson-induced hyperemia to some degree. In subjects receiving aminophylline, coronary blood flow fell below 2fold of baseline within $<1 \mathrm{~min}$, whereas subjects not receiving aminophylline remained above 2-fold of baseline for an additional $7 \mathrm{~min}$. Notably, at the 10-minute mark, neither group of subjects returned to baseline perfusion [23].

In our subjects, we suspect that persistent hyperemia occurred in the post-regadenoson recovery phase due to residual vasodilatory effects of the drug, given its relatively long terminal half-life [23]. Although regadenoson has a relatively weak affinity for the $A_{2 A}$ receptor [29], such that its peak vasodilatory effect is brief, drug that is distributed throughout the tissues may return to the blood compartment during recovery and cause a low level of coronary vasodilation. While aminophylline seems to help alleviate the side effects of regadenoson, and is therefore often used as an antidote, its ability to reverse the coronary vasodilatory effects of regadenoson has not been fully described. In our subjects, aminophylline did not eliminate the vasodilatory effects of regadenoson - that is, some degree of hyperemia persisted at recovery in most subjects who received aminophylline. Our results are consistent with those of the previously mentioned catheterization study, in which intracoronary blood flow was measured invasively after regadenoson administration; although the average peak blood flow velocity was reduced by aminophylline, it did not return to baseline by the 10-minute mark [23]. A potential explanation for these observations is that theophylline has a low affinity for the $\mathrm{A}_{2 \mathrm{~A}}$ receptor [30], so it may dissociate relatively rapidly, allowing some of the residual regadenoson to rebind post-stress. Interestingly, our aminophylline group had a significantly greater decline in heart rate from stress to recovery, suggesting that aminophylline is somewhat active at the $\mathrm{A}_{2 \mathrm{~A}}$ receptor at 15- 
minute follow up. Regardless, our results suggest that the order of the study protocol is critically important if perfusion reserve is to be determined by regadenoson CMR or, similarly, by any other myocardial perfusion imaging modality.

Although the rest-stress protocol must be used to quantify perfusion reserve accurately, it is unknown if this protocol would lower the sensitivity of regadenoson CMR for detecting ischemic perfusion defects. Administering a lower contrast dose at rest, in a manner similar to reststress SPECT imaging, or allowing a prolonged delay between rest and stress imaging, might help mitigate this problem. An alternative strategy would consist of a 2-day protocol with stress imaging performed on the first day and rest imaging performed on a subsequent day. The rest portion could be eliminated if obvious perfusion defects were present on the initial stress images. Of course, such an approach would be costly and time-consuming and would require clinical validation to justify widespread use.

Perhaps our most noteworthy finding is that several patients demonstrated more hyperemia at recovery than at stress. In these subjects, recovery-to-rest ratios (MPRirecov) were similar to stress-to-rest ratios (MPRi-rest) in the remaining subjects, suggesting that some individuals have a delayed maximal hyperemic response to regadenoson. Aminophylline appears to have little impact on this effect. Although all these subjects had an increase in perfusion from rest to stress, as would be expected, mean MPRi-rest and MPRi-recov were significantly lower than in subjects with rapid hyperemia. Interestingly, the proportions of delayed-hyperemia patients in the aminophylline and non-aminophylline groups were similar, leading us to believe that this phenomenon is unlikely to have biased our overall results. Although patients with delayed hyperemia tended to have slightly higher diastolic blood pressures, no values were pathologically elevated, so this is unlikely to be a clinically useful metric. Further studies will be needed to identify potential predictors of and explanations for the delayed-hyperemic response.

The persistence of hyperemia beyond the immediate post-stress period may raise the question of whether patients with flow-limiting coronary stenoses can safely receive regadenoson without prolonged post-procedural monitoring - i.e., whether such patients might still be "ischemic" when discharged from the CMR center immediately after scan completion. However, it is important to note that regadenoson, like adenosine, does not typically cause true ischemia, but rather a relative lack of hyperemia in segments subtended by diseased vessels. Although it is possible that regadenoson causes a prolonged discrepancy in myocardial blood flow between territories supplied by healthy and diseased arteries, it is unlikely that this results in adverse clinical effects, based on the drug's excellent safety record in the SPECT literature [16].

\section{Limitations}

This study was conducted in a small number of healthy volunteers. However, this number was sufficient to answer the questions we posed with confidence, as confirmed by statistical analysis. Based on common practice with adenosine CMR, we performed recovery imaging 15 minutes post-stress. It is possible that postregadenoson recovery imaging either earlier (i.e., 23 min after aminophylline administration) or later (20$30 \mathrm{~min}$ after stress) would have demonstrated a lesser degree of residual hyperemia. As all of our subjects had normal perfusion, we could not assess whether a reststress protocol, as opposed to a stress-recovery protocol, would mask the presence of ischemia due to contrast contamination of stress images. Five individuals who received a very low dose of contrast $(0.025 \mathrm{mmol} / \mathrm{kg})$ were included in the analysis; however, their MPRi-rest was not significantly different from the other subjects. Finally, it is unknown whether a relatively modest (approximately 20\%) underestimation of perfusion reserve, as seen with MPRi-recov in patients receiving aminophylline, would have clinical significance. Future studies are needed to answer this question.

\section{Conclusions}

We have demonstrated that a regadenoson CMR stressrecovery protocol underestimates perfusion reserve compared to a rest-stress protocol. Therefore, for reliable quantification of myocardial perfusion reserve, the latter approach is preferable. Further research will be needed to determine whether a rest-stress protocol would lower the sensitivity of regadenoson CMR for detecting ischemic perfusion defects, and to establish the length of time between rest and stress imaging that would be necessary to eliminate this issue.

We additionally showed that some subjects have a delayed hyperemic response during the supposed recovery phase; future studies are needed to elucidate the clinical relevance and underlying explanation for this finding.

\section{Abbreviations \\ CMR: Cardiac magnetic resonance; MPRi: Myocardial perfusion reserve index; MPRi-rest: Up-slope ratio of stress to rest perfusion; MPRi-recov: Up-slope ratio of stress to recovery perfusion; AV: Atrioventricular; MPR: Myocardial perfusion reserve; SSFP: Steady-state free precession; LV: Left ventricle; LGE: Late gadolinium enhancement; LVEF: Left ventricular ejection fraction; SPECT: Single photon emission computed tomography.}

\section{Competing interests}

This study was supported by a grant from Astellas Pharma.

\section{Authors' contributions}

NB acquired and analyzed the majority of the image datasets, performed all statistical analyses, and drafted the manuscript. BF was involved in study design and image acquisition and analysis. CY was involved in image analysis. DK provided expertise regarding the pharmacokinetics and pharmacodynamics of regadenoson. KD was involved in image acquisition. $\mathrm{RL}$ and VM provided assistance with manuscript revision. AP conceived of the study and was involved in image acquisition and analysis and manuscript revision. All authors read and approved the final manuscript. 


\section{Disclosures}

Research grant from Astellas Pharma.

Received: 6 September 2012 Accepted: 18 December 2012 Published: 28 December 2012

\section{References}

1. Greenwood JP, Younger JF, Brown JM, Everett CC, Bijsterveld P, Ridgway JP, Radjenovic A, Dickinson CJ, Ball SG, Plein S. Cardiovascular magnetic resonance and single-photon emission computed tomography for diagnosis of coronary heart disease (CE-MARC): a prospective trial. Lancet 2012, 379:453-60.

2. Nandalur KR, Dwamena BA, Choudhri AF, Nandalur MR, Carlos RC. Diagnostic performance of stress cardiac magnetic resonance imaging in the detection of coronary artery disease: a meta-analysis. J Am Coll Cardiol 2007, 50:1343-53.

3. Klem I, Heitner JF, Shah DJ, Sketch MH, Behar V, Weinsaft J, Cawley P, Parker M, Elliott M, Judd RM, Kim RJ. Improved detection of coronary artery disease by stress perfusion cardiovascular magnetic resonance with the use of delayed enhancement infarction imaging. J Am Coll Cardiol 2006, 47:1630-8.

4. Pilz G, Bernhardt P, Klos M, Ali E, Wild M, Hofling B. Clinical implication of adenosine-stress cardiac magnetic resonance imaging as potential gatekeeper to invasive examination in patients with AHA/ACC class II indication for coronary angiography. Clin Res Cardiol 2006, 95:531-8.

5. Plein S, Greenwood JP, Ridgway JP, Cranny G, Ball SG, Sivananthan MU. Assessment of non-ST-segment elevation acute coronary syndromes with cardiac magnetic resonance imaging. J Am Coll Cardiol 2004, 44:2173-81.

6. Plein S, Radjenovic A, Barmby D, Greenwood JP, Ball SG, Sivananthan MU. Coronary artery disease: myocardial perfusion MR imaging with sensitivity encoding versus conventional angiography. Radiology 2005, 235:423-30

7. Schwitter J, Wacker CM, van Rossum AC, Lombardi M, Al-Saadi N, Ahlstrom H, Dill T, Larsson HBW, Flamm SD, Marquardt M, Johansson L. MR-IMPACT: comparison of perfusion-cardiac magnetic resonance with single-photon emission computed tomography for the detection of coronary artery disease in a multicentre, multivendor, randomized trial. Eur Heart J 2008, 29:480-9.

8. Wong DTL, Leung MCH, Das R, Liew GYH, Dundon BK, Molaee P, Teo KSL, Meredith IT, Worthley MI, Worthley SG. Diagnostic accuracy of adenosine stress cardiovascular magnetic resonance following acute ST-segment elevation myocardial infarction post primary angioplasty. J Cardiovasc Magn Reson. 2011; 13:62.

9. Paetsch I, Jahnke C, Wahl A, Gebker R, Neuss M, Fleck E, Nagel E. Comparison of dobutamine stress magnetic resonance, adenosine stress magnetic resonance, and adenosine stress magnetic resonance perfusion. Circulation 2004, 110:835-42.

10. Jahnke C, Nagel E, Gebker R, Kokocinski T, Kelle S, Manka R, Fleck E, Paetsch I. Prognostic value of cardiac magnetic resonance stress tests: adenosine stress perfusion and dobutamine stress wall motion imaging. Circulation 2007, 115:1769-76.

11. Ingkanisorn WP, Kwong RY, Bohme NS, Geller NL, Rhoads KL, Dyke CK, Paterson I, Syed MA, Aletras AH, Arai AE: Prognosis of negative adenosine stress magnetic resonance in patients presenting to an emergency department with chest pain. J Am Coll Cardiol 2006, 47:1427-32.

12. Bodi V, Husser O, Sanchis J, Nunez J, Monmeneu JV, Lopez-Lereu MP, Bosch MJ, Rumiz E, Minana G, Garcia C, Diago JL, Chaustre F, Moratal D, Gomez C, Aguilar J, Chorro FJ, Llacer A. Prognostic implications of dipyridamole cardiac MR imaging: a prospective multicenter registry. Radiology 2012, 262:91-100

13. Voigtlander T, Schmermund A, Bramlage P, Elsasser A, Magedanz A, Kauczor $\mathrm{HU}$, Mohrs OK. The adverse events and hemodynamic effects of adenosine-based cardiac MRI. Korean J Radiol 2011, 12:424-30.

14. Khoo JP, Grundy BJ, Steadman CD, Sonnex EP, Coulden RA, McCann GP. Stress cardiovascular magnetic resonance in routine clinical practice: referral patterns, accuracy, tolerance, safety and incidental findings. $\mathrm{Br}$ 」 Radiol 2012, 85:e851-7.

15. Hendel RC, Bateman TM, Cerqueira MD, Iskandrian AE, Leppo JA, Blackburn B, Mahmarian JJ. Initial clinical experience with regadenoson, a novel selective A2A agonist for pharmacologic stress single-photon computed tomography myocardial perfusion imaging. J Am Coll Cardiol 2005, 46:2069-75.
16. Al Jaroudi W, Iskandrian AE: Regadenoson: a new myocardial stress agent. J Am Coll Cardiol 2009, 54:1123-30.

17. Freed BH, Turner KM, Yodwut C, Tarroni G, Estep E, Bhave NM, Narang A, Tanaka S, Corsi C, Gayat E, Czobor P, Cavanaugh K, Lang R, Mor-Avi V, Patel AR. Regadenoson cardiovascular magnetic resonance myocardial perfusion imaging predicts need for future revascularization [abstract]. J Cardiovasc Magn Reson. 2012; 14(Suppl 1):P7.

18. Patel AR, Antkowiak PF, Nandalur KR, West AM, Salerno M, Arora V, Christopher J, Epstein FH, Kramer CM. Assessment of advanced coronary artery disease: advantages of quantitative cardiac magnetic resonance perfusion analysis. J Am Coll Cardiol 2010, 56:561-9.

19. DiBella EVR, Fluckiger JU, Chen L, Kim TH, Pack NA, Matthews B, Adluru G, Priester T, Kuppahally S, Jiji R, McGann C, Litwin SE. The effect of obesity on regadenoson-induced myocardial hyperemia: a quantitative magnetic resonance imaging study. Int J Cardiovasc Imaging 2012, 28:1435-44.

20. Greenwood JP, Maredia N, Radjenovic A, Brown JM, Nixon J, Farrin AJ, Dickinson C, Younger JF, Ridgway JP, Sculpher M, Ball SG, Plein S. Clinical evaluation of magnetic resonance imaging in coronary heart disease: the CE-MARC study. Trials 2009, 10:62.

21. Schwitter J, Wacker CM, Wilke N, Al-Saadi N, Sauer E, Huettle K, Schonberg SO, Luchner A, Strohm O, Ahlstrom H, Dill T, Hoebel N, Simor T, MR-IMPACT Investigators. MR-IMPACT II: magnetic resonance imaging for myocardial perfusion assessment in coronary artery disease trial: perfusion-cardiac magnetic resonance vs. single-photon emission computed tomography for the detection of coronary artery disease: a comparative multicentre, multivendor trial. Eur Heart J 2012, Epub ahead of print.

22. Gordi T, Frohna P, Sun HL, Wolff A, Belardinelli L, Lieu H. A population pharmacokinetic/pharmacodynamic analysis of regadenoson, a selective A2A-receptor agonist, in healthy male volunteers. Clin Pharmacokinet 2006, 45:1201-12.

23. Lieu HD, Shryock JC, von Mering GO, Gordi T, Blackburn B, et al.: Regadenoson, a selective A2A adenosine receptor agonist, causes dose-dependent increases in coronary blood flow velocity in humans. J Nuc Cardiol 2007, 14:514-20.

24. Lockie T, Ishida M, Perera D, Chiribiri A, De Silva K, Kozerke S, Marber M, Nagel E, Rezavi R, Redwood S, Plein S. High-resolution magnetic resonance myocardial perfusion imaging at 3.0-Tesla to detect hemodynamically significant coronary stenoses as determined by fractional flow reserve. J Am Coll Cardiol 2011, 57:70-5.

25. Wohrle J, Nusser T, Merkle N, Kestler HA, Grebe OC, Marx N, Hoher M, Kochs $M$, Hombach V. Myocardial perfusion reserve in cardiovascular magnetic resonance: Correlation to coronary microvascular dysfunction. J Cardiovasc Magn Reson 2006, 8:781-7.

26. Shivu GN, Phan TT, Abozguia K, Ahmed I, Wagenmakers A, Henning A Narendran P, Stevens M, Frenneaux M. Relationship between coronary microvascular dysfunction and cardiac energetics impairment in type 1 diabetes mellitus. Circulation 2010, 121:1209-15.

27. Nakajima H, Onishi K, Kurita T, Ishida M, Nagata M, Kitagawa K, Dohi K, Nakamura M, Sakuma H, Ito M. Hypertension impairs myocardial blood perfusion reserve in subjects without regional myocardial ischemia. Hypertens Res 2010, 33:1144-9.

28. Patel AR, Epstein FH, Kramer CM. Evaluation of the microcirculation: advances in cardiac magnetic resonance perfusion imaging. J NuCl Cardiol 2008, 15:698-708.

29. Gao Z, Li Z, Baker SP, Lasley RD, Meyer S, Elzein E, Palle V, Zablocki JA, Blackburn $B$, Belardinelli L. Novel short-acting A2A adenosine receptor agonists for coronary vasodilation: inverse relationship between affinity and duration of action of A2A agonists. J Pharmacol Exp Ther 2001, 298:209-18.

30. Ongini E, Fredholm BB: Pharmacology of adenosine A2A receptors. Trends Pharmacol Sci 1996, 17:364-72.

\section{doi:10.1186/1532-429X-14-89}

Cite this article as: Bhave et al: Considerations when measuring myocardial perfusion reserve by cardiovascular magnetic resonance using regadenoson. Journal of Cardiovascular Magnetic Resonance 2012 14:89. 